\title{
Esforços Alterados Sobre os Dentes e sua Influência na Memória (Estudo em Humanos)
}

Rejane Nunes* Eleutério Araújo Martins* *

\begin{abstract}
RESUMO
Trinta estudantes, jovens e saudáveis, de Odontologia, foram submetidos, uma vez ao dia, durante três dias consecutivos, (intervalos de 24 horas) a dois testes comuns de memória: 1 - o reconhecimento de faces famosas em fotografias,

e 2 - a identificação do ano em que eventos importantes ocorreram.

Os estudantes foram divididos em três grupos de dez estudantes cada um, e submetidos a ambos os testes acima mencionados sempre o teste de número

1 , precedendo o teste de número 2.

No primeiro dia, logo após os testes terem sido aplicados pela primeira vez, um dos grupos (Grupo C) foi passivo (todos os seus integrantes) de aplicação de uma tira de borracha, simulando uma força ortodôntica (orthodontic-like force

- OLF), entre o primeiro e o segundo pré-molares superiores direitos.

No segundo dia, uma hora antes da aplicação dos testes, outro grupo (Grupo

B) foi passível de uma experiência inusitada (NE - novel experience) ao permanecer por 10 minutos em um ambiente onde nenhum de seus componentes tinha estado anteriormente. Os testes foram aplicados da mesma forma e ordem que no primeiro dia, em intervalos de 24 horas, no segundo e terceiro dias a todos os três grupos. (Grupo A - controle).

Os resultados obtidos mostraram resultados melhorados estatisticamente significativos no teste de reconhecimento de faces famosas para o Grupo $B(N E)$ tanto no segundo dia $-\mathrm{P}<0.01$, como no terceiro dia $-\mathrm{P}<0.05$.

Os resultados obtidos na aplicação do teste de eventos significativos mostrou resultados melhorados estatisticamente significativos tanto no Grupo $B$ (NE) como no Grupo $C(O L F)$ onde $P<0.01$, mas apenas no terceiro dia.

Os resultados indicam que ambas as diferenças avaliadas - experiência inusitada e esforços alterados sobre os dentes - podem melhorar o desempenho de humanos

em testes de memória, sugerindo o envolvimento em ambos os casos de mecanismos neuronais endógenos que modulam a percepção da dor e a memória.
\end{abstract}

\footnotetext{
*Professora Auxiliar de Ensino - Discipllnas de Oclusão FO - UFRGS.

**Professor Titular - Disciplinas de

Oclusão FO - UFRGS.
} 


\section{SUMMARY}

Thirty young healthy students of dentistry were submmited, once a day, on three consecutive days (24 hours interval) to two simple memoty tests: 1 - the recognition of famous faces in pictures, and 2 - the recall of the year major events took place.

The students were divided in three groups of ten students each, and submitted to both tests listed above, allways the test number one before the test number two.

On the first day, just after the tests were applied for the first time, one of the groups (GROUP C) was submitted to the application of a rubber band, simulating an orthodontic force (orthodontic-like force-OLF) between the first and second upper rigth bicuspids, of all the ten components.

On the second day, one hour before the tests, one group (GROUP B) was exposed to a novel experience, (NE) staying for 10 minutes in a room they had never been before. The tests were made in the same way and order both in the second and the third day for all three groups. GROUP A was used as a control group.

The results obtained showed statistically significant enhanced scores in the

famous faces test, for the GROUP $B(N E)$ both in the second day $(P<0.01)$ and in the third day $(P<0.05)$.

The results obtained from the application of the major events test demonstrated statistically significant enhanced scores in both Group B (NE) and Group C (OLF) $-P<0.01$, but only in the third day.

The results seem to indicate that both situations induced - novel experience and orthodontic-like force - can enhance the performance of humans in simple memory tests, suggesting the involvement of neuronal endogenous mechanisms that are known to modulate pain perception and memory.

\section{DESCRITORES}

Oclusão dentária; Ortodontia; Memória; Neuroquímica.

\section{INTRODUÇÃO}

Influências sobre a memória de fatores tais como: ingestão de drogas, ingestão de álcool, liberação de hormônios, exposição à situações inusitadas e/ou mudanças no meio ambiente e até mesmo alterações nos momentos de treinamento e testes, tem sido relatadas, nos últimos cinco anos de maneira alentada na literatura (1-16).

O sistema estomatognático, por ser uma das importantes vias de comunicação entre humanos e de manifestação comportamental é também passível de disfunções quando da presença de alterações emocionais e, por isso, tem sido objeto de atenção de pesquisadores, principalmente com relação a situações de raiva, medo, tensão (17), e de dor e seus mecanismos de modulação $(18,19)$. Atualmente há consenso em que a estimulação direta da substância cinzenta periaquedutal (PAG), inibe neurônios nociceptivos no subnúcleo caudalis bem como que a estimulação de mecanorreceptores de baixo limiar, no campo periférico, também inibe a resposta de neurônios caudalis, tanto nociceptivos específicos (NS) como de raio de ação dinâmica ampla (WDR), donde se pode concluir que a modulação da dor pode resultar tanto de estimulação das fibras periféricas sensoriais periféricas não-nociceptivas como de estimulação de regiōes centrais do cérebro (20). Está comprovado que respostas sensoriais a estímulos da polpa dentária, são rapidamente suprimidos durante 250 a até $1.000 \mathrm{~ms}$. por estimulação da substância cinzenta periaquedutal e do núcleo rafe magnus (NRM) e que a serotonina parece ser 0 principal neurotransmissor ativo nesse mecanismo inibitório (19). Em termos clínicos, o efeito modulador da estimulação cutânea, é conhecido desde a antigüidade. Tanto ser otonina como dinorfina são liberadas quando nervos cutâneos superficiais são estimulados 
(ex.: TENS) e seu efeito não é reversivel por naloxone. Esses estímulos estão inseridos na categoria de estimulos cutâneos não dolorosos (20).

A descoberta das endorfinas revelou a presença inata de um sistema endógeno antinociceptivo, o qual normalmente modula a dor. Dor aguda causa liberação de opióides endógenos no fluido cérebro-espinal (CSF) e na corrente sangüínea. A ação da encefalina é muito curta, durando apenas uns poucos segundos. A ação da beta-endorfina na corrente sangüínea, retardada por alguns minutos, dura períodos bem mais longos. A ação das endorfinas é parcial, senão totalmente interrompida por naloxone, um antagonista da morfina (20). Assim, qualquer ação moduladora que seja significativamente inibida ou interrompida por naloxone, deve envolver o sistema antinociceptivo endorfínico, como descrito em alguns trabalhos de investigação da memória $(12,13)$. Algumas dessas substâncias químicas (encefalina, beta-endorfina) são lideradas quando ocorrem al terações determinadas por esforços oclusais modificados experimentalmente, em humanos (21). Parece haver uma interrelação entre dor sustentada, naloxone e sua influência no sistema antinociceptivo endógeno (20).

Trabalhos sobre interferências oclusais e tensão, provocando alterações missíveis na quantidade excretada de adrenalina na urina (21), bem como significativas modificações das concentrações de metionina-encefalina na polpa humana, em dentes previamente sujeitos à forças constantes, indicam que, pelo menos, uma trajetória neuropeptidérgica é mobilizada nesses casos (22). Outrossim, também foram identificados sistemas descendentes inibitórios da dor, envolvendo a liberação de neurotransmissores como a noroepinefrina e a serotonina, que podem ser ativados por alterações tensionais (23).

Parece ser razoável imaginar que disfunções que ocorrem no sistema estomatognático e que, comprovadamente, determinam o aparecimento de sintomatologia variada possam também interferir em um ou mais dos mecanismos endógenos admitidos como tendo influência na modulação da memória, em humanos.

\section{OBJETIVOS}

Uma revisão bibliográfica atualizada motivou a indagação, e assim este estudo foi dirigido no sentido de verificar, em humanos, se a aplicação de esforços alterados em um ou mais dentes, e que se sabe, provocam sintomas dolorosos variáveis em estruturas periodontais, musculares e articulares (24), determinaria alterações no desempenho em testes de memória. Simultaneamente foram repetidas experiências anteriores em humanos (13), utilizando a exposição à uma experiência inusitada (NE) para permitir uma comparação entre resultados.

\section{MATERIAIS E MÉTODOS}

Foram selecionados trinta estudantes de Odontologia da FOUFRGS, todos com boa saúde, ausência de no máximo um elemento dentário, que não tivessem história prévia de problemas oclusais e situados na faixa etária entre 18 e 22 anos. Os estudantes foram divididos em três grupos de 10 estudantes cada um, e todos foram submetidos a dois testes comuns de memória. Os testes foram aplicados pelo mesmo investigador de maneira a que apresentassem um grau de dificuldade semelhante em tempo e linguagem. A aplicação dos testes foi sempre feita num mesmo ambiente, e que era perfeitamente conhecido de todos (laboratório). Os testes utilizados foram: 1 - um teste de faces famosas, e 2 - um teste de rememoração do ano em que eventos importantes ocorreram.

No teste de faces famosas, cada um dos participantes era solcitado a identificar as faces de pessoas famosas (artistas, políticos esportistas) através da observação de foto- grafias coloridas tiradas de revistas com grande distribuição nacional. No teste de eventos importantes, era solicitado aos participantes que lembrassem do ano em que os mesmos tinham ocorrido. Os eventos foram selecionados entre aqueles grandemente difundidos pela imprensa nos últimos cinco anos (a morte de Tancredo Neves, quando um time de futebol gaúcho foi campeão mundial interclubes, etc.).

No teste de faces famosas cada um dos participantes era apresentado a 10 (dez) faces diferentes, em fotografias. As faces nunca foram repetidas em dias subseqüentes e foram selecionadas de tal maneira a permitir uma distribuição harmônica entre classes representadas. Para as respostas corretas eram atribuídos dois (2) pontos; para as respostas parcialmente corretas (foi um presidente mas não sei o nome) era atribuído um (1) ponto e zero (0) pontos para as respostas erradas. Isto permi tia um escore máximo de 20 (vinte) pontos no teste, a cada dia.

No teste de eventos importantes, a cada dia eram selecionados eventos diferentes de um total de mais de cem elaborados pelos investigadores. Para as respostas corretas eram atribuídos três (3) pontos; erros de mais ou menos um (1) ano recebiam dois (2) pontos; erros com variação de mais ou menos (5) anos recebiam um (1) ponto e erros maiores ou falta de resposta, zero (0) pontos. Isto permitia um escore máximo de trinta (30) pontos a cada teste diário.

Nos três dias em que os testes foram aplicados, o teste de faces famosas sempre precedeu o teste de eventos importantes.

No primeiro dia, logo após os três grupos terem sido submetidos aos testes, todos os componentes do Grupo C (OLF) foram alvo de aplicação de uma banda elástica de borracha, de consistência e espessura igual em todos os casos, entre os primeiros e segundos pré-molares superiores direitos, simulando a ação de uma força ortodôntica, o que foi mantido por 48 horas.

No segundo dia -23 horas após 
os primeiros testes, e uma hora antes dos segundos testes - o grupo B foi levado para o sub-solo da Faculdade onde estava ligado o compressor de ar central da mesma e em cujas dependências nenhum deles havia estado antes (a entrada é vedada). Os membros do grupo não sabiam da finalidade de sua estada no ambiente, onde eles permaneceram por dez (10) minutos. Na vigésima quarta hora, após os primeiros testes, todos os três grupos C, (OLF) - $B$, (experiência nova) e $A$, (controle) foram submetidos novamente aos testes, o que tornou a ocorrer no terceiro dia, 24 horas após.

\section{RESULTADOS}

Todos os escores obtidos pelos participantes foram registrados e submetidos a análise estatística por pesquisador credenciado.*

A observação da Tabela de número 1 permite que se vejam os resultados obtidos pelos três grupos quando submetidos aos testes de faces famosas nos três dias em que durou a experiência. Pode se notar que apenas um grupo, o grupo B (exposto a uma experiência inusitada) apresentou diferença estatisticamente significativa na melhoria dos escores obtidos em teste feito 1 hora após a experiência $(P<0.01)$ bem como no teste feito 24 horas após a mesma $(P<0.05)$.

$\mathrm{Na}$ tabela de número 2 podem ser observados os resultados apresentados pelos três grupos quando submetidos aos testes de eventos importantes nos três dias do trabaIho. Não apenas o grupo B, mas também o grupo $C$, no terceiro dia, obtiveram escores com diferenças estatisticamente significativas daquelas obtidas no primeiro dia de testes. Os escores obtidos nos testes realizados no segundo dia não mostra-

\footnotetext{
* Nosso reconhecimento ao Prof. Edgar Mário Wagner, Professor de Bio-Estatistíca do Instituto de Blociências pela colaboração altamente valiosa na análise de resultados.
}

\section{TABELA 1}

\begin{tabular}{|c|c|c|c|c|}
\hline & 1: DIA & 2. DIA & 3. DIA & \\
\hline GRUPO & $\begin{array}{c}0 \text { horas } \\
\text { MÉDIA — D }\end{array}$ & $\begin{array}{c}24 \text { horas } \\
\text { MÉDIA — DP }\end{array}$ & $\begin{array}{c}48 \text { horas } \\
\text { MÉDIA — DP }\end{array}$ & $\begin{array}{l}\text { DIFERENÇA } \\
\text { SIGNIFICATIVA } \\
\text { ENTRE MÉDIAS }\end{array}$ \\
\hline $\begin{array}{c}\text { A } \\
\text { CONTROLE }\end{array}$ & $14,6 \pm 3,4$ & $15,4 \pm 3,2$ & $15,8 \pm 3,9$ & N.S. \\
\hline $\begin{array}{c}\text { B } \\
\text { EXPERIÊNCIA } \\
\text { INUSITADA }\end{array}$ & $14,8 \pm 2,9$ & $17,3 \pm 1,9$ & $16,3 \pm 2,8$ & $\begin{array}{l}0-24 h .^{* *} \\
0-48 \text { h.* }\end{array}$ \\
\hline $\begin{array}{c}\text { C } \\
\text { FORÇAS } \\
\text { ANORMAIS }\end{array}$ & $16,3 \pm 2,3$ & $17,0 \pm 2,5$ & $17,8 \pm 1,8$ & N.S. \\
\hline
\end{tabular}

N.S. = Diferença não significativa $-P>0,05$.

${ }^{*}, * *=$ Diferença significativa para: $P<0,05$ e $P<0,01$

Teste de identificação de faces de pessoas famosas

(Escore máximo $=20$ pontos)

\section{TABELA 2}

\begin{tabular}{|c|c|c|c|c|}
\hline & 1: DIA & 2. DIA & 3. DIA & \\
\hline GRUPO & $\begin{array}{c}0 \text { horas } \\
\text { MÉDIA } \pm D\end{array}$ & $\begin{array}{c}24 \text { horas } \\
\text { MÉDIA } \pm \text { DP }\end{array}$ & $\begin{array}{c}48 \text { horas } \\
\text { MÉDIA — DP }\end{array}$ & $\begin{array}{l}\text { DIFERENÇA } \\
\text { SIGNIFICATIVA } \\
\text { ENTRE MÉDIAS }\end{array}$ \\
\hline $\begin{array}{c}\text { A } \\
\text { CONTROLE }\end{array}$ & $12,6 \pm 4,7$ & $13,4 \pm 4,1$ & $13,8 \pm 5,7$ & N.S. \\
\hline $\begin{array}{c}\text { B } \\
\text { EXPERIÊNCIA } \\
\text { INUSITADA }\end{array}$ & $11,3 \pm 2,9$ & $13,3 \pm 2,8$ & $16,2 \pm 6,0$ & $\begin{array}{c}0-48 \text { h. }^{* *} \\
\left(A_{1}-A_{3}\right)\end{array}$ \\
\hline $\begin{array}{c}\text { C } \\
\text { FORÇAS } \\
\text { ANORMAIS }\end{array}$ & $13,5+5,7$ & $15,6+5,5$ & $17,5+5,6$ & $\begin{array}{c}0-48 h_{. * *}^{*} \\
\left(A_{1}-A_{3}\right)\end{array}$ \\
\hline
\end{tabular}

N.S. = Diferença não significativa * $=$ Diferença significativa para $-P<0,01$

Teste de rememoraçăo de datas de eventos

(Escore máximo $=30$ pontos)

ram diferenças estatisticamente significativas daqueles do primeiro dia. Ambos os grupos - $\mathrm{B}$ e $\mathrm{C}$ - apresentaram uma diferença de $P<0.01$.

O Grupo A, usado como controle não mostrou diferenças estatisticamente significativas em nenhum dos dias e em nenhum dos testes aplicados.

\section{DISCUSSÃO}

Os resultados obtidos parecem confirmar resultados prévios de ou- tros autores de que uma experiência inusitada pode alterar o desempenho, em testes de memória, em humanos (13). Entretanto, eles também sugerem que, ao menos nas condições em que o presente trabalho foi realizado e com o tipo de pacientes utilizados, os resultados podem ser influenciados pela modalidade do teste e podem sofrer alterações 48 horas após à exposição (NE).

Os resultados obtidos pelo grupo $C$, submetido a esforços alterados sobre os dentes e sua comparação com aqueles obtidos pelo grupo B 
(experiência nova) quando da utilização de testes de eventos importantes, indicam que, provavelmente uma ou mais das substâncias liberadas como decorrência de forças constantes ou intermitentes, alteradas, sobre os dentes, e identificadas por alguns autores $(21,22)$, podem determinar reações semelhantes, ou mesmo serem idênticas, aquelas relacionadas em investigações anteriores, comprovadamente influenciando a memória, em humanos (3, $4,5,6)$.

\section{CONCLUSÕES}

Os resultados obtidos, nas condições experimentais do presente trabalho, indicam que:

1. A aplicação de forças alteradas a nivel de dentes, em humanos, pode modificar suas respostas a testes de memória, 48 horas após;

2. Mecanismos que envolvem a liberação de substâncias endógenas devem estar envolvidos, similarmente ao que ocorre quando humanos são submetidos a uma experiência inusitada;

3. Forças similares às utilizadas em tratamentos ortodônticos, podem provocar reações bem mais amplas do que aquelas normalmente admitidas em dentes e estruturas anexas.

\section{REFERÊNCIAS BIBLIOGRÁFICAS}

1. IZQUIERDO, I. Memory consolidation: not a useful hypothesis in search for memoryenhancing drugs. Trends Pharmacol. Sci., 7:476-7, 1986.

2. NETTO, C.C.; DIAS, R.D.; IZQUIERDO, I. Interaction between consecutive learnings: inhibitory avoidance and habituation. Behav. Neural Biol., 44:515-20, 1985.

3. McGAUGH, J.L. Peripheral and central adrenergic influences on brain systems involved in the modulation of memory storage. Ann. NY. Acad. Sci., 444:150-61, 1985.

4. IZQUIERDO, I. \& NETTO, C.A. Factors that influence retrieval measured 0,3 , or 6 hours after inhibitory avoidance training. Behav. Neural Biol., 43:260-73, 1985.

5. IZQUERDO, I. Opioid-cathecolamine and opioid-cholinergic interactions in memory regulation. Trends Pharmacol. Sci., 6:192-3, 1985.

6. IZQUIERDO, I.; NETTO, C.A.; DALMAZ, C.; CHAVES, M.L.; PEREIRA, M.; SIEGFRIED, B. Construction and reconstruction of memories. Braz. J. Med. Biol. Res., $21: 9-25,1988$.

7. GOLD, P.E. \& ZORNETZER, S.F. The mnemon and its juices: neuro-modulation of memory processes. Behav. Neural Biol., $38: 151-89,1983$.

8. IZQUIERDO, I. \& DIAS, R.D. Memory as a state dependent phenomenon: role of ACTH and epinephrine. Behav. Neural Biol. 38:144-9, 1983.

9. NETTO, C.A.; DIAS, R.D.; IZQUIERDO, I. Training in an open field: simultaneous leaming of habituation and of a water finding task, and differential effect of posttraining naloxone, B-endorphin, leu-enkephalin and electroconvulsive shock. Psychoneuroendocrinology, 11:437-46, 1986.

10. IZQUIERDO, I. \& NETTO, C.A. Role of B-endorphin in behavioral regulation. Ann. NY. Acad. Sci. 444:162-77, 1985.

11. NETTO, A.C.; OLIVEIRA, C.B.; GIANLUPI, A.; QUILLFELDT, J.; IZQUIGDO, I. Pretest administration of B-endorphin, or of electro-convulsive shock reversas the memory, disruptive effect of posttraining electroconvulsive shock. Peptides, 8:605-8, 1987.

12. IZQUIERDO, I. \& McGAUGH, J.L. Effect of a novel experience prior to training or testing on retention of an inhibitory avoidance task in mice: involvement of an opioid system. Behav. Neural Biol., 44:228-38, 1985.

13. CHAVES, M.L.F. \& IZQUIERDO, I. Previous exposure to a novel experience enhances performance in two simple memory tests, in humans. Braz. J. Med. Biol. Res., 19:211-9, 1986.

14. PEREIRA M.E.; DALMAZ, C.; ROSAT, R. M.; IZQUIERDO, I. Diazepam blocks the interfering effect of posttraining behavioral manipulation on retention of a shuttle avoidance task. Psychopharmacology, 94:402-4, 1988.

15. IZQUERDO, I. Endogenous state-at, dency: memory depends on the relation between the neurohumoral and nonal states present after training and at ine time of testing. IN: LYNCH, G.; McGAUGH, J.L.; WEINBERGER, N.M.; GUILFORD, N.Y. Eds. Neurobiology of learming and memory. New York, Academic Press, 1984. p.333-50.

16. CAHILL, L.; BRIONI, J.; IZQUIERDO, I. Retrograde memory enhancement by diazepam: its relation to anterograde amnesia and some clinical implications. Psychophar macology, 90:554-6, 1986.

17. EVASKUS, D.S. \& LASKIN, D.M. A biomechanical measure of stress in patients : "? myofascial pain dysfunction syndrome. $J$. Dent. Res., 51:1464-6, 1972.

18. SESSLE, B.J. \& HU, J.W. Raphe inducec supression of the jaw-opening reflex and single neurons in trigeminal subnucleus oralis, and influence of naloxone and subnucleus caudalis. Pain, 10:19-36, 1981.

19. SESSLE, B.J. The neurobiology of facial and dental pain: present knowledge future directions. J. Dent. Res., 66:962-81, 1987.

20. BELL, W. Oro facial pains: classification, diagnosis, management. W. Chicago, Year Book Medical Publishers, 1989. 448p., p.31, p.56-7.

21. KOBAYASHI, Y.; TAKEDA, Y.; ISHIHA$R A, H$. The influence of experimental occlusal interference on psychoendocrine responses. J. Dent. Res., 64:746, 1985.

22. WALKER, J.A.; TANZER, F.S.; HARRIS, E.F.; WAKELYN, C.; DESIDERIO, D.M. The enkephalin response in human tetth to orthodontic force. Am.J. Orthod. Dentofacial Orthop., 92:9-16, 1987.

23. DUBNER, R. Recent advances in our understanding of pain. In: KLINEBERG, ' \& SESSLE, B.J.; Eds., Oro-facial pain and neuromuscular dysfunction. Oxford, Pergamon Press, 1985. 229p., p.4-17.

24. NUNES, R. \& MARTINS, E.A. Trauma oclusal artificialmente provocado: singis $e$ sintomas no sistema estomatognático Rev. Gaucha Odontol. In press. 